\title{
Risk factors associated with pulmonary arterial hypertension in patients with systemic sclerosis and implications for screening
}

\author{
C.P. Denton* and E. Hachulla ${ }^{\#}$
}

ABSTRACT: Pulmonary arterial hypertension (PAH) is a relatively common complication of systemic sclerosis (SSc) affecting $5-12 \%$ of patients, and its development is associated with significant morbidity and a particularly poor prognosis. Deaths associated with other complications of SSc, such as renal crisis, have fallen significantly in recent years in line with improvements in their treatment and management. However, mortality due to PAH in this population, although improved, has shown a less dramatic decline.

The early diagnosis of PAH in SSc would allow for earlier treatment, before functional and haemodynamic impairment becomes severe; this may further improve outcome, and evidence suggests that screening of SSc patients for PAH is associated with improved survival.

In addition, patients with PAH associated with SSc are not a homogeneous population and they differ in terms of disease haemodynamic severity, functional capacity and rate of disease progression. Likewise, management strategies may differ, and the ability to stratify patients may help optimise screening and treatment. A number of patient-, clinical- and disease-specific risk factors associated with the development and prognosis of PAH in SSc have been identified, but their optimal use, alone or in combination, in screening and stratification of patients remains to be established.

KEYWORDS: Pulmonary arterial hypertension, screening, systemic sclerosis

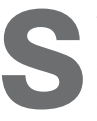
ystemic sclerosis (SSc) is associated with a range of organ complications, including renal disease, cardiac manifestations, pulmonary fibrosis and pulmonary arterial hypertension (PAH). All such complications impact on survival in patients with SSc, but this is most marked in patients who develop PAH (fig. 1) [1]. Recent advances in the treatment of complications such as scleroderma renal crisis have improved survival in patients with SSc, particularly those with the more severe diffuse cutaneous subtype (dcSSc) [1-3]. Mortality from scleroderma renal crisis fell markedly between the early 1970s and 2000s due to the use of angiotensin converting enzyme inhibitors [1-2]. However, there was little apparent improvement in mortality associated with $\mathrm{PAH}$, thus, it has become one of the leading causes of mortality in SSc patients, accounting for over a quarter of all SScrelated deaths $[2,4,5]$.
Overall, the prevalence of PAH in SSc reported in recent studies ranges between $5 \%$ and $12 \%$, with evidence of an increase in rate of diagnosis in line with increased disease awareness and improvements in screening [1, 6-9]. In the Royal Free Hospital cohort (London, UK), the frequency of PAH in SSc in both dcSSc and the limited cutaneous subgroup (lcSSc) began to increase from $\sim 2$ yrs after diagnosis, increasing to $\sim 5 \%$ and $6 \%$, respectively, after 5 yrs and $13 \%$ and $15 \%$, respectively, after 10 yrs (unpublished data). The incidence of PAH in SSc was estimated to be 0.61 cases per 100 patient-yrs based on a 3-yr followup of patients included in the French ItinérAIRSclérodermie study [10]. Although it has been generally considered that $\mathrm{PAH}$ is associated with lcSSc [11], recent evidence has shown that PAH is almost as frequent in patients with dcSSc [1, 12]. While organ complications in dcSSc tend to occur relatively early in the disease course, with the
AFFILIATIONS

*Royal Free Campus, UCL Medical School, London, UK.

${ }^{\#}$ Claude Huriez Hospital, Dept of Internal Medicine, National

Scleroderma Centre, Lille University, Lille, France.

CORRESPONDENCE

C.P. Denton

Centre for Rheumatology and

Connective Tissue Diseases

Dept of Inflammation

Division of Medicine

UCL Medical School

Royal Free Campus

Rowland Hill Street

London

NW3 2PF

UK

E-mail: cdenton@medsch.ucl.ac.uk

Received:

Aug 122011

Accepted:

Aug 302011

PROVENANCE

Publication of this peer-reviewed article was supported by Actelion Pharmaceuticals Ltd, Switzerland (principal sponsor, European Respiratory Review issue 122). 


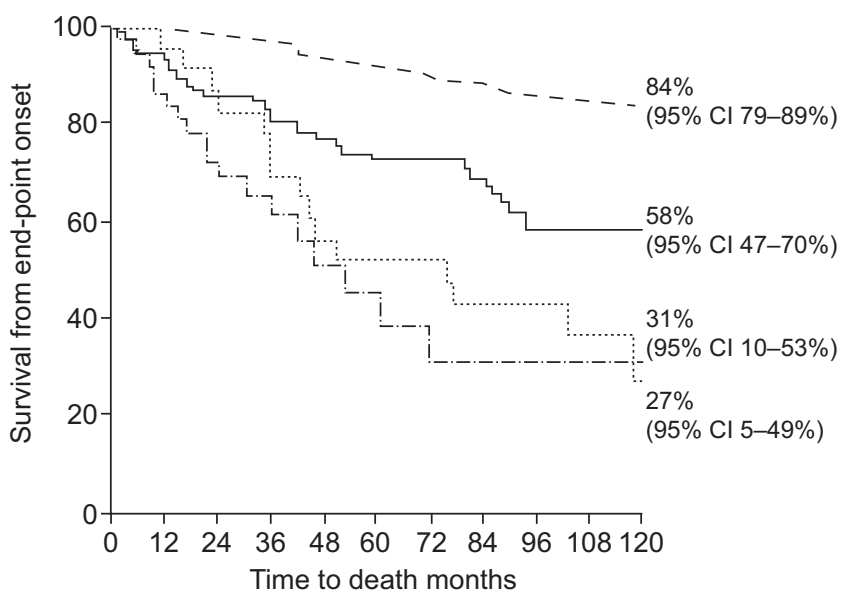

Subjects at risk $\mathrm{n}$

$\begin{array}{lccc}\text { No } & 247 & 222 & 175 \\ \text { PF } & 90 & 49 & 19 \\ \text { PAH } & 38 & 7 & 0 \\ \text { PF+PH } & 23 & 12 & 3\end{array}$

FIGURE 1. Impact of complications on survival in systemic sclerosis. - - - -: no complications; —_: pulmonary fibrosis (PF); …: PF plus pulmonary hypertension $(\mathrm{PH})$; -..--: pulmonary arterial hypertension $(\mathrm{PAH}) . \mathrm{p}<0.001$. Modified from [1].

majority arising within 3 yrs of diagnosis [13], such complications tend to develop later in lcSSc patients. However, although PAH has been reported to be a late complication of lcSSc [11], around half of such patients have early-onset PAH, arising within the first 5 yrs following diagnosis of SSc [14].

$\mathrm{PAH}$ associated with SSc (PAH-SSc) has a particularly poor prognosis. If untreated, 1-yr survival after diagnosis is significantly shorter in patients with PAH-SSc compared with patients with idiopathic PAH (IPAH) [15]. In the ItinérAIRSclérodermie cohort, the 3-yr survival estimate of 91.1\% in SSc patients was reduced to $56.3 \%$ in those who had $\mathrm{PAH}$ at baseline [16]. Long-term survival is generally highest in $\mathrm{PAH}$ patients who have less severe disease at diagnosis (World Health Organization functional class (WHO FC) I/II versus II/ IV) $[17,18]$, and studies have shown that treatment with disease-specific therapies early in the course of PAH may improve long-term outcome [19, 20]. Given the significant incidence of PAH in patients with SSc and the high mortality associated with this complication if untreated, there is a clear need for early detection before patients have marked clinical and haemodynamic deterioration, and for timely treatment.

The diagnosis of PAH can be challenging, especially in the early stages and, particularly, in patients with diseases that have multiple potential causes of dyspnoea, the primary presenting symptom in PAH, such as SSc. As a result, detection of PAH in SSc is often delayed, and patients are only diagnosed when they have advanced disease with severe clinical and haemodynamic impairment [21-23]. Annual screening for PAH in symptomatic patients with SSc is recommended in recent guidelines [24, 25], although the cost-effectiveness of screening for PAH in asymptomatic SSc patients has been questioned [25]. However, recent data suggest that screening of asymptomatic SSc patients is associated with improved survival [1,9]. Identification of risk factors for and early markers of PAH in SSc, and the development and implementation of an optimal screening programme, would allow for early detection and treatment of this complication, and may result in further improvements in morbidity and mortality for these patients.

\section{RISK FACTORS AND RISK STRATIFICATION}

SSc patients with "early $\mathrm{PAH}^{\prime}$ are a heterogeneous population $[17,18]$. For example, some patients have relatively stable disease with mild symptoms and well-preserved exercise capacity, but despite this they may have severe haemodynamic impairment. Due to the lack of specific symptoms, such patients are often diagnosed relatively late in terms of disease course and so may have developed a high degree of pulmonary vascular disease. Other groups include patients identified early in the disease course when they are mildly symptomatic but who have a progressive disease type, with mild $\mathrm{PAH}$ with a low degree of pulmonary vascular disease and slowly progressive disease, and patients with early PAH as well as coexisting pulmonary fibrosis or pulmonary veno-oclusive disease (PVOD). In the latter group, differential diagnosis of PVOD presents a particular challenge [26]. It would be useful to identify risk factors associated with each of these groups, and to be able to stratify patients for screening and management purposes, as this may differ.

A number of patient-, clinical- and disease-specific factors that may be associated with the development of PAH in SSc have been identified. In general, there are conflicting data regarding the link between patient-specific factors and the likelihood of $\mathrm{PAH}$ in SSc. For example, older age has been identified as a risk factor in some studies $[8,27]$, possibly due to the effect of disease duration or of older age at onset of SSc, both of which have been shown to be risk factors for PAH-SSc [8, 28]. However, this link has not been shown in all studies [29]. In females, the risk of PAH-SSc has been reported to be increased during the postmenopausal period and linked with a decrease in the protective effects of oestrogen on the endothelium [30, 31]. Clinical factors linked to PAH development include the degree of skin involvement and more severe peripheral vascular disease (Raynaud's phenomenon and digital ulcers), as well as the presence of pulmonary fibrosis, microstomia, gastro-oesophageal reflux and dysphagia, although not all studies have found the same associations [8, 29, 32, 33]. In general, PAH has been considered to be a late complication, predominantly affecting patients with lcSSc; however, as discussed previously, lcSSc patients can have early-onset PAH [14] and PAH is almost as frequent in patients with dcSSc [1, 12].

Diffusing capacity of the lung for carbon monoxide $(D \mathrm{~L}, \mathrm{CO})$ has been shown to be a strong predictor of survival in PAH [34], and may also predict the development of PAH-SSc [32]. A decrease in DL,CO in patients with lcSSc was reported to be a strong predictor of the occurrence of $\mathrm{PAH} \geqslant 10$ yrs later; patients who developed PAH had a significantly lower mean $D \mathrm{~L}, \mathrm{CO}$ (52\% predicted) almost 5 yrs before diagnosis compared with closely matched controls who did not develop PAH (52\% versus $81 \%$ pred) [32]. In a more recent study, a decrease in the $D \mathrm{~L}, \mathrm{CO} /$ alveolar volume $(\mathrm{VA})$ ratio $<70 \%$ pred was shown to be a significant predictor of PAH-SSc over a median 29 months of follow-up (hazard ratio 18.81; $p=0.014$ ) [35]. The level of serum $\mathrm{N}$-terminal pro-brain natriuretic peptide (NT-proBNP) reflects the severity of right ventricular dysfunction and PAH [17, 35-38]. 
Baseline and serial changes in NT-proBNP levels have been shown to be highly predictive of survival $[37,39]$ and of response to therapy [37, 40]. In SSc, high NT-proBNP levels (>97th percentile of normal) identified those patients who went on to develop PAH during a median 29-month follow-up with a sensitivity of $90 \%$, specificity of $90.3 \%$, positive predictive value of $69.2 \%$ and negative predictive value of $96 \%$ [35]. This study also found that, while both an elevated baseline NT-proBNP level and a $D \mathrm{~L}, \mathrm{CO} / V \mathrm{~A}$ ratio $<60 \%$ and $<70 \%$ were predictive of the development of PAH, the combination of $D \mathrm{~L}, \mathrm{CO} / \mathrm{VA}<70 \%$ plus high NT-proBNP was highly predictive (HR 47.2), with a sensitivity and specificity of $75 \%$ and $97 \%$, respectively [35]. The identification of such risk factors for the development of PAH raises the possibility of risk stratification of patients to further refine and improve screening, and the early detection and treatment of this complication in SSc. However, currently identified risk factors require validation and further investigation to confirm their prognostic importance and their potential value, alone or in combination, in such stratification.

\section{SCREENING FOR PAH IN SSc}

Although screening for $\mathrm{PAH}$ is widely recommended in symptomatic patients with SSc [24, 25], there has been less agreement regarding screening for $\mathrm{PAH}$ in asymptomatic
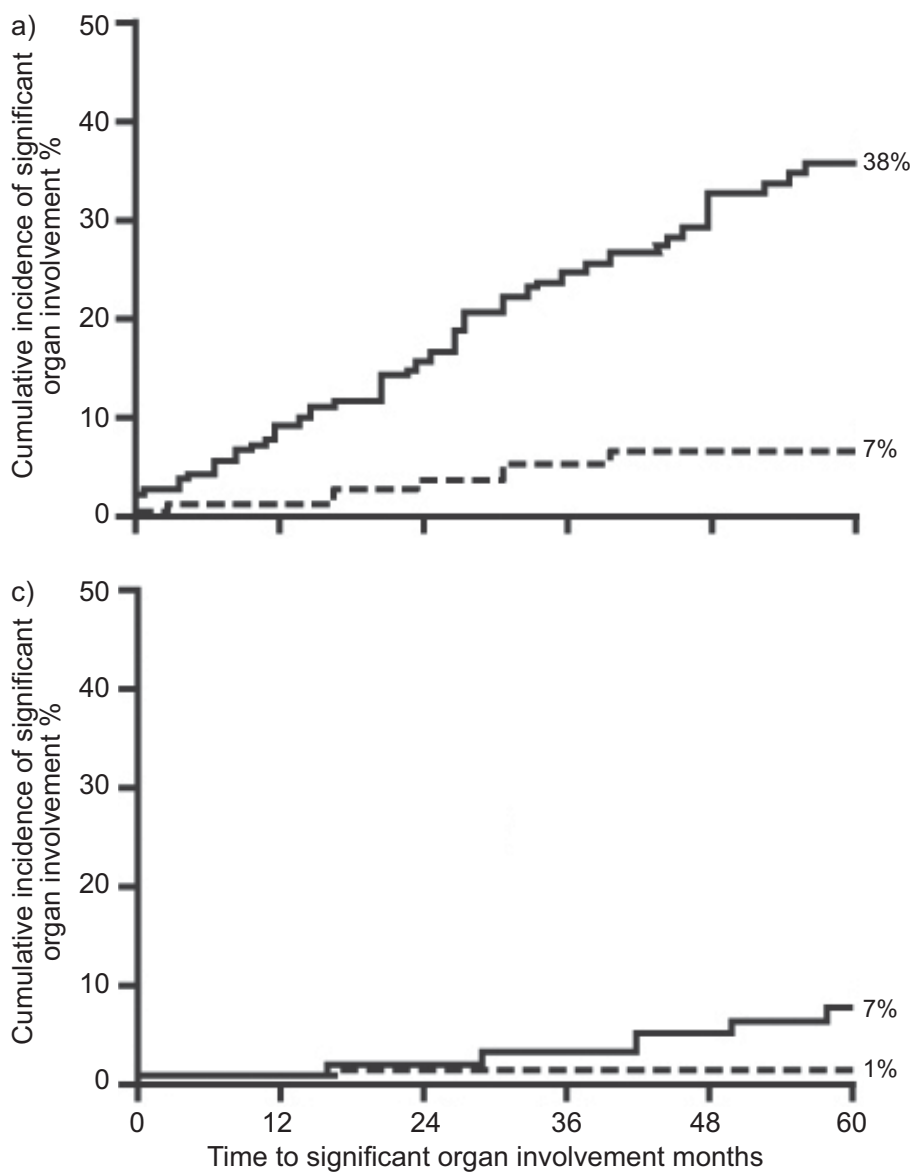

patients. For example, whereas regular screening of asymptomatic SSc patients is recommended by the American College of Cardiology Foundation/American Heart Association guidelines [24], the European Society of Cardiology/European Respiratory Society guidelines state that the cost-effectiveness of this procedure has not been clarified compared with symptom-based screening [25]. Given that $>10 \%$ of patients with SSc go on to develop PAH, screening for this complication would appear to be warranted, and there are an increasing number of studies that support this strategy. The frequency of diagnosis of both $\mathrm{PAH}$ and pulmonary fibrosis was found to be markedly increased in a recent study comparing a contemporary cohort of patients with disease onset between 2000 and 2003 and a historical cohort with disease onset between 1990 and 1993 [1]. In association with this, survival in patients with dcSSc was significantly higher in the contemporary cohort, which the authors concluded was a result of better and more complete ascertainment of lung complications due to the use of systematic annual screening (fig. 2) [1]. A comparison of two cohorts of patients from the same management era (2000-2003) found that those who were diagnosed with $\mathrm{PAH}$ during a systemic $\mathrm{PAH}$ detection programme ("detected" cohort) had significantly higher 3-, 5- and 8-yr survival rate compared with those diagnosed during routine clinical practice ("routine" cohort)

b)

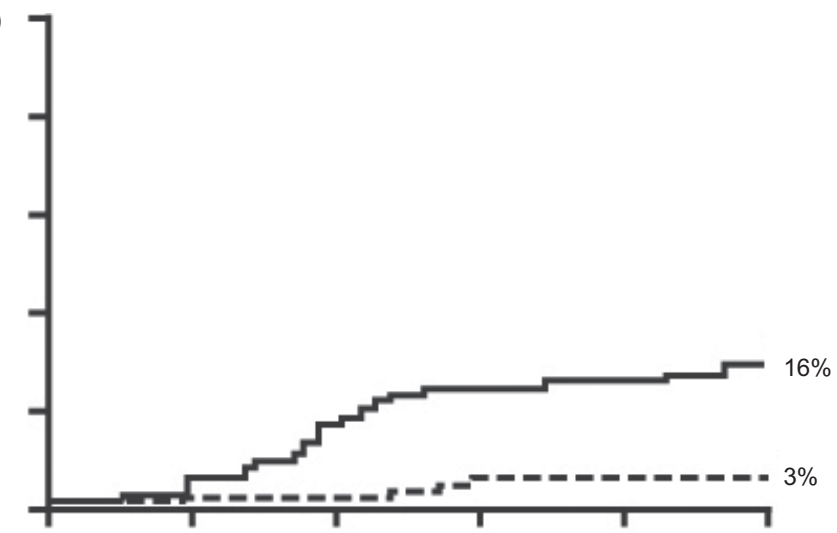

d)

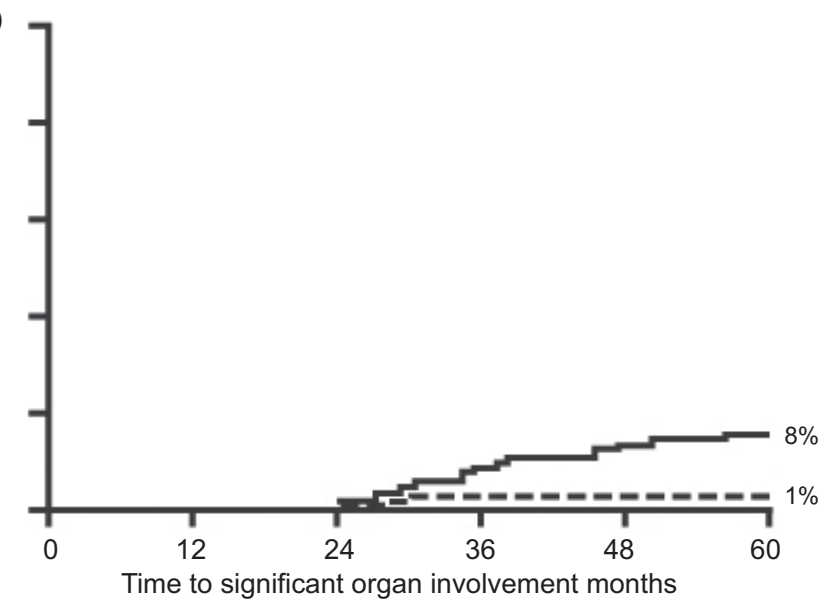

FIGURE 2. Impact of regular screening on ascertainment of a, b) pulmonary fibrosis (PF) and c, d) pulmonary arterial hypertension (PAH) in systemic sclerosis (SSc). Kaplan-Meier estimation of cumulative incidence of PF and PAH in patients from a contemporary (screened) and historical (unscreened) cohort in two SSc subsets: a, c) diffuse cutaneous SSc and b, d) limited cutaneous SSc. — - disease onset, 2000-2003; - - - disease onset, 1990-1993. a, b) $p<0.001, c) p=0.148, d) p=0.002$. Reproduced from [1] with permission from the publisher. 
(81\%, $73 \%$ and $64 \%$ versus $31 \%, 25 \%$ and $17 \%$, respectively; $\mathrm{p}=0.0037$ ) [9]. In addition, detected patients had less advanced disease (lower WHO FC), and better haemodynamics (lower mean pulmonary arterial pressure $(P \mathrm{pa})$ and pulmonary vascular resistance (PVR), and higher cardiac output) compared with the routine cohort [9]. This study used echocardiography to screen SSc patients over a 3-yr period, with those with a peak tricuspid regurgitation (TR) jet velocity of $>3 \mathrm{~m} \cdot \mathrm{s}^{-1}$ or $2.8-3 \mathrm{~m} \cdot \mathrm{s}^{-1}$ and unexplained dyspnoea being referred for right heart catheterisation. However, optimal screening tools and methodology are, as yet, unknown, and this is confounded by a lack of consensus over which patients to screen [41].

Right heart catheterisation is the standard confirmatory test for PAH, which is defined by a mean $P$ pa $\geqslant 25 \mathrm{mmHg}$ together with a pulmonary capillary wedge pressure $\leqslant 15 \mathrm{mmHg}$ [25]. However, right heart catheterisation is underused outside of highly specialised centres, and the clinical utility of the technique as a screening tool is limited by its invasive nature. Therefore, other methodologies are required for accurate, routine screening. Echocardiography can be used to provide estimates of key right heart haemodynamic parameters, such as $P$ pa. Although a lower cut-off of estimated systolic Ppa $(P$ pa,syst $) \leqslant 36 \mathrm{mmHg}$ suggests that PAH is unlikely and a higher cut-off of $>50 \mathrm{mmHg}$ suggests it is likely, there remains a "grey area" using echocardiography-calculated $P$ pa alone. Guidelines state that estimation of $P$ pa based on echocardiography is not suitable for screening in cases of mild, asymptomatic pulmonary hypertension [25], although recent studies suggest that echocardiography-calculated Ppa may in fact be useful in identifying early-stage $\mathrm{PAH}$ as part of a screening regimen [9]. Although less directly linked to the accepted haemodynamic definition of $\mathrm{PAH}$ (i.e. $\mathrm{Ppa} \geqslant 25 \mathrm{mmHg}$ ), TR velocity has been shown to be a useful screening parameter in SSc. In the ItinérAIR-Sclérodermie study [10], patients with a peak TR velocity of $>3 \mathrm{~m} \cdot \mathrm{s}^{-1}$ or $2.5-3 \mathrm{~m} \cdot \mathrm{s}^{-1}$ and unexplained dyspnoea at screening were referred for right heart catheterisation; this method identified 33 patients with suspected $\mathrm{PAH}$ out of a cohort of 599, 18 (55\%) of whom were found to have $\mathrm{PAH}$ on catheterisation [7]. The proportion of patients (15 $(45 \%)$ out of 33 ) who were subsequently found not to have $\mathrm{PAH}$ on right heart catheterisation (false-positives) in this study, and so who underwent an "unnecessary" invasive technique, emphasises an important consideration in the development of screening methods. Combining several echocardiography parameters may improve its accuracy and usefulness as a screening tool. Combining echocardiography-derived $P$ pa,syst $\geqslant 35 \mathrm{mmHg}$ with right ventricular Tei-index $>0.36$ was shown to improve the accuracy of PAH-SSc detection as confirmed by right heart catheterisation from $80 \%$ to $90 \%$ [42]. Evaluation of echocardiography-derived $\mathrm{Ppa}$, post-systolic tricuspid annular movement (TAPSE) as a marker of right ventricular function, and the acceleration time of right outflow as an indirect marker of PVR suggested early-stage PAH in 19 patients with normal lung volumes, no significant pulmonary fibrsois and mildly reduced $D \mathrm{~L}, \mathrm{CO}$ (mean \pm SD $71 \pm 18 \%$ of theoretical), which was subsequently confirmed by right heart catheterisation in $15(79 \%)$ patients [43].

Pulmonary function testing may also be useful in screening for $\mathrm{PAH}$ in SSc. Patients with PAH usually have decreased DL,CO (typically in the range of $40-80 \%$ pred) and mild-to-moderate reductions in lung volume [25]. DL,CO together with arterial oxygen saturation measured by pulse oximetry have recently been used to derive a pulmonary function formula which may help select those patients at risk of $\mathrm{PAH}$ who require right heart catheterisation [44]. A risk prediction score has also been recently proposed to identify patients at risk of developing $\mathrm{PAH}$, based on age, forced vital capacity and $D \mathrm{~L}, \mathrm{CO} / V \mathrm{~A}$ ratio [45]. A decreased $D L, C O / V A$ ratio has been shown to be a predictor of $\mathrm{PAH}$ in SSc [35], and a decrease in DL,CO may predict the occurrence of pulmonary hypertension several years later in SSc patients without significant interstitial lung disease [32]. However, although DL,CO does seem to be an important predictor of the potential occurrence of $\mathrm{PAH}$ and mortality in patients who develop PAH [34], a cut-off value which completely excludes the possibility of PAH has yet to be defined [6]. Furthermore, DL,CO does not appear to correlate closely with haemodynamic variables $[6,46]$, and does not independently predict the presence of pulmonary hypertension [47].

Serum levels of a number of biomarkers have been shown to be related to the extent of right ventricular dysfunction and to correlate with haemodynamic, echocardiographic and functional measurements of pulmonary vascular disease. Raised NT-proBNP levels have been found to be directly related to the severity of $\mathrm{PAH}$ and, in screening programmes, a cut-off value of $395 \mathrm{pg} \cdot \mathrm{mL}^{-1}$ had a positive predictive value of $95 \%$ and a negative predictive value of $56.5 \%$ for the presence of PAH-SSc [37]. Similarly, a cut-off value of $239.4 \mathrm{pg} \cdot \mathrm{mL}^{-1}$ identified PAH in SSc patients with a sensitivity of $45 \%$ and specificity of $90 \%$ in a recent study by CAVAGNA et al. [48], although the authors note that normal levels of NT-proBNP did not exclude the possibility of PAH in SSc. Interestingly, NT-proBNP levels have been reported to be significantly higher in PAH-SSc compared with $\mathrm{IPAH}$, despite less severe haemodynamic impairment, and more closely related to haemodynamics in PAH-SSc than IPAH [39], and also to be highest in patients with PAH-SSc relative to patients with $\mathrm{PAH}$ associated with other connective tissue diseases [49].

A number of auto-antibodies specific to SSc have been reported to be associated with, although not specific to, $\mathrm{PAH}$, including anti-centromere antibody [50], anti-U3 ribonucleoprotein [51] and anti-Th/To [52]. The characterisation of antibody profiles may be helpful in the assessment and prognosis of patients with SSc, but a role in screening for PAH remains to be established. Recently, differences in gene expression in peripheral blood mononuclear cells between lcSSc patients with and without associated PAH have been described, increasing the prospect of using gene expression profiles as biomarkers to predict the risk of PAH in these patients $[53,54]$.

Although potentially valuable, none of the tools mentioned have been shown to accurately identify all patients with $\mathrm{PAH}-$ SSc used alone, and optimal threshold values for various parameters remain to be fully established. Perhaps unsurprisingly, there is evidence to suggest that combining results from a number of screening methods may better detect PAH than any single measure alone. KHANNA et al. [55] found that an echocardiographic right ventricular systolic pressure $(P$ pa,syst in the absence of pulmonary obstruction) $>50 \mathrm{mmHg}$ had the greatest discriminatory power to detect $\mathrm{PAH}$ in a cohort of SSc patients "at risk" of or diagnosed with $\mathrm{PAH}$, detecting $\sim 40 \%$ 
of patients with right heart catheterisation confirmed PAH. However, adding serum NT-proBNP $>100 \mathrm{pg} \cdot \mathrm{mL}^{-1}$ and $D \mathrm{~L}, \mathrm{CO}$ $<60 \%$ of expected to the echocardiography criterion identified an additional $27 \%$ of patients with PAH [55].

Ideally, the identification of an accurate screening test using a single method or combination of noninvasive methods would decrease the number of unnecessary right heart catheterisations (that is, reduce the false-positive fraction) and increase the true-positive fraction, or the sensitivity, of the screening test for an early and accurate diagnosis of PAH in SSc patients. Although a number of potential tools are available, further supportive data for their use alone and in combination as part of a screening algorithm are required.

\section{DETECT STUDY}

The DETECT (Early, Simple and Reliable Detection of Pulmonary Arterial Hypertension in Systemic Sclerosis) study, which was initiated in 2008, is a prospective, observational, cohort study in two stages consisting of a cross-sectional stage (baseline) and a 3-yr longitudinal follow-up (fig. 3) [56]. The objective of DETECT is to evaluate a range of accessible, noninvasive screening tools and clinical findings (electrocardiography, echocardiography, serum biomarkers, pulmonary function testing, 6-min walk test, Borg dyspnoea index, medical history, patient demographics and disease characteristics) for their ability to detect pulmonary hypertension in SSc patients, and to identify simple screening methods to support earlier, reliable detection of this complication. With a target recruitment of 500 patients across 91 sites, the study primarily aims to determine the discriminatory and predictive ability of single screening tests for PAH in SSc patients compared with right heart catheterisation and single or multiple tests for screening of PAH in SSc patients. DETECT will also allow for the investigation of a range of secondary and exploratory objectives in this large prospective SSc cohort, including the incidence of PAH and pulmonary hypertension in SSc over the 3-yr follow-up period, the correlation between change in screening test results and change in measures of clinical status, and the association of potential prognostic or risk factors (e.g. potential biomarkers such as endothelin-1 and anti-nuclear antibodies, etc.) with the

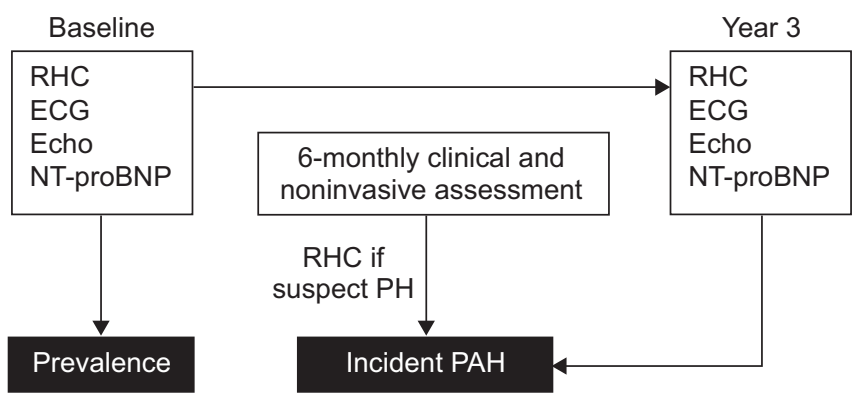

FIGURE 3. Schematic diagram of the DETECT study [56]. Inclusion criteria: systemic sclerosis or mixed connective tissue disease for $\geqslant 3 \mathrm{yrs}$; diffusing capacity of the lung for carbon monoxide $<60 \%$ predicted; and no known pulmonary hypertension $(\mathrm{PH})$ or left heart disease. Study commenced October 2008; cross-sectional data analysis 2011; longitudinal phase predicted completion end 2013. RHC: right heart catheterisation; ECG: electrocardiogram; Echo: echocardiography; NT-proBNP: N-terminal pro-brain natriuretic peptide; $\mathrm{PAH}$ : pulmonary arterial hypertension. development of PAH and pulmonary hypertension. Although results from this study will undoubtedly be valuable, there are some caveats for their application to the wider SSc population. The study population for DETECT is restricted to SSc patients with a $D \mathrm{~L}, \mathrm{CO}<60 \%$ of expected and who are, therefore, at high risk of developing PAH. Moreover, inclusion criteria state that patients must have SSc disease duration of $>3$ yrs from onset of first non-Raynaud's symptom. As previously discussed, pulmonary hypertension can be an early complication in a subgroup of patients with SSc [14], which may mean that there are prevalent cases included in the first part of the DETECT study and these could differ from incident cases identified later. Despite this, however, the identification of single or multiple noninvasive screening tests for the early detection of PAH in SSc patients by DETECT would have a significant influence on patient management and would be expected to impact on standards of care in a variety of clinical settings beyond SSc.

\section{IMPORTANCE OF EARLY DETECTION}

$\mathrm{PAH}$ in patients with $\mathrm{SSc}$ is particularly aggressive and disease progression can be rapid, therefore, delays in the diagnosis and treatment of PAH-SSc may adversely affect survival. The early diagnosis of such complications is therefore of major importance; however, early detection is only relevant if similarly early treatment is associated with significant clinical benefit. The majority of studies showing the benefits of PAH-targeted therapies have been carried out in patients with more advanced disease (WHO FC III and IV), although the limited number of studies investigating patients with less advanced disease have shown that early treatment, when clinical and haemodynamic impairment are less severe, is associated with improved outcome $[19,20]$. For example, following 3 months of epoprostenol therapy, patients with IPAH in WHO FC I or II had a significantly higher probability of survival than those in FC III or IV, with 1- and 3-yr survival rates of $100 \%$ and $88 \%$, and $77 \%$ and $33 \%$, respectively [19]. Currently, the only randomised, placebo-controlled trial dedicated to FC II patients is the EARLY study, in which $\sim 10 \%$ of patients had PAH-SSc [20]. Treatment with bosentan was associated with improvements in haemodynamics and prevention of clinical deterioration relative to placebo in this patient group, and results from this study led to regulatory approval for bosentan in WHO FC II. The placebo arm of the EARLY study also showed that, if left untreated, patients with mildly symptomatic PAH can show both clinical and haemodynamic deterioration, despite maintaining their exercise capacity, emphasising the importance of early intervention recommended by treatment guidelines [20]. However, registry data show that, without screening, only $\sim 16 \%$ of PAHSSc patients are diagnosed in WHO FC II [18]; the vast majority are not diagnosed until their disease is more advanced when prognosis is poorer and there is a clear need to improve this situation.

\section{CONCLUSIONS}

Patients with SSc are at a high risk of developing PAH, and this is associated with significant morbidity and particularly poor prognosis. The identification of markers that would allow for the stratification of patients based on the degree of this risk may allow for tailoring of screening and management strategies. However, although a number of risk factors have been identified, their suitability for such stratification remains to be established. 
Screening of SSc patients for PAH is recommended by treatment guidelines and has been shown to be associated with improved survival. Early diagnosis before haemodynamic impairment becomes marked allows for earlier treatment using PAH-specific therapies which may further improve outcome. The detection of $\mathrm{PAH}-\mathrm{SSc}$ in its earliest stages is particularly complicated in SSc patients. Although a number of screening tools are available, their usefulness in an optimal screening algorithm alone or in combination remains to be established. The DETECT study will provide prospective data with which to quantify and evaluate risk factors and screening tools for $\mathrm{PAH}$ in SSc, thus allowing optimisation of screening in this important population.

\section{STATEMENT OF INTEREST}

C.P. Denton has received research grant funding and acted as a consultant to Actelion Pharmaceuticals, Pfizer, GlaxoSmithKline and Roche. He has also received lecture fees from these companies. E. Hachulla has received fees for speaking from Actelion, Pfizer and GlaxoSmithKline; funds for research from Pfizer; and fees for consulting from Actelion, Pfizer, GlaxoSmithKline and United Therapeutics.

\section{ACKNOWLEDGEMENTS}

We received editorial assistance from L. Thomas (Elements Communications Ltd, Westerham, UK), supported by Actelion Pharmaceuticals Ltd (Allschwil, Switzerland).

\section{REFERENCES}

1 Nihtyanova SI, Tang EC, Coghlan JG, et al. Improved survival in systemic sclerosis is associated with better ascertainment of internal organ disease: a retrospective cohort study. QJM 2010; 103: 109-115.

2 Steen VD, Medsger TA. Changes in causes of death in systemic sclerosis, 1972-2002. Ann Rheum Dis 2007; 66: 940-944.

3 Mouthon L, Bérezné A, Bussone G, et al. Scleroderma renal crisis: a rare but severe complication of systemic sclerosis. Clin Rev Allergy Immunol 2011; 40: 84-91.

4 Steen VD, Medsger TA Jr. Long-term outcomes of scleroderma renal crisis. Ann Intern Med 2000; 133: 600-603.

5 Tyndall AJ, Bannert B, Vonk M, et al. Causes and risk factors for death in systemic sclerosis: a study from the EULAR Scleroderma Trials and Research (EUSTAR) database. Ann Rheum Dis 2010; 69: 1809-1815.

6 Mukerjee D, St George D, Knight C, et al. Echocardiography and pulmonary function as screening tests for pulmonary arterial hypertension in systemic sclerosis. Rheumatology (Oxford) 2004; 43: 461-466.

7 Hachulla E, Gressin V, Guillevin L, et al. Early detection of pulmonary arterial hypertension in systemic sclerosis: a French nationwide prospective multicenter study. Arthritis Rheum 2005; 52: 3792-3800.

8 Avouac J, Airò P, Meune C, et al. Prevalence of pulmonary hypertension in systemic sclerosis in European Caucasians and metaanalysis of 5 studies. J Rheumatol 2010; 37: 2290-2298.

9 Humbert M, Yaici A, de Groote P, et al. Screening for pulmonary arterial hypertension in patients with systemic sclerosis: clinical characteristics at diagnosis and long-term survival. Arthritis Rheum 2011; [Epub ahead of print DOI:10.1002/art.30541].

10 Hachulla E, de Groote P, Gressin V, et al. The three-year incidence of pulmonary arterial hypertension associated with systemic sclerosis in a multicenter nationwide longitudinal study in France. Arthritis Rheum 2009; 60: 1831-1839.

11 Cox SR, Walker JG, Coleman M, et al. Isolated pulmonary hypertension in scleroderma. Intern Med J 2005; 35: 28-33.
12 Hunzelmann N, Genth E, Krieg T, et al. The registry of the German Network for Systemic Scleroderma: frequency of disease subsets and patterns of organ involvement. Rheumatology (Oxford) 2008; 47: 1185-1192.

13 Shand L, Lunt M, Nihtyanova S, et al. Relationship between change in skin score and disease outcome in diffuse cutaneous systemic sclerosis: application of a latent linear trajectory model. Arthritis Rheum 2007; 56: 2422-2431.

14 Hachulla E, Launay D, Mouthon L, et al. Is pulmonary arterial hypertension really a late complication of systemic sclerosis? Chest 2009; 136: 1211-1219.

15 Kawut SM, Taichman DB, Archer-Chicko CL, et al. Hemodynamics and survival in patients with pulmonary arterial hypertension related to systemic sclerosis. Chest 2003; 123: 344-350.

16 Hachulla E, Carpentier P, Gressin V, et al. Risk factors for death and the 3-year survival of patients with systemic sclerosis: the French ItinérAIR-Sclérodermie study. Rheumatology (Oxford) 2009; 48: 304-308.

17 Condliffe R, Kiely DG, Peacock AJ, et al. Connective tissue diseaseassociated pulmonary arterial hypertension in the modern treatment era. Am J Respir Crit Care Med 2009; 179: 151-157.

18 Hachulla E, Launay D, Yaici A, et al. Pulmonary arterial hypertension associated with systemic sclerosis in patients with functional class II dyspnoea: mild symptoms but severe outcome. Rheumatology (Oxford) 2010; 49: 940-944.

19 Sitbon $\mathrm{O}$, Humbert $\mathrm{M}$, Nunes $\mathrm{H}$, et al. Long-term intravenous epoprostenol infusion in primary pulmonary hypertension: prognostic factors and survival. I Am Coll Cardiol 2002; 40: 780-788.

20 Galiè N, Rubin LJ, Hoeper M, et al. Treatment of patients with mildly symptomatic pulmonary arterial hypertension with bosen$\tan$ (EARLY study): a double-blind, randomised controlled trial. Lancet 2008; 371: 2093-2100.

21 Le Pavec J, Humbert M, Mouthon L, et al. Systemic sclerosisassociated pulmonary arterial hypertension. Am J Respir Crit Care Med 2010; 181: 1285-1293.

22 Humbert M, Sitbon O, Chaouat A, et al. Pulmonary arterial hypertension in France: results from a national registry. Am J Respir Crit Care Med 2006; 173: 1023-1030.

23 Badesch DB, Raskob GE, Elliott CG, et al. Pulmonary arterial hypertension: baseline characteristics from the REVEAL Registry. Chest 2010; 137: 376-387.

24 McLaughlin VV, Archer SL, Badesch DB, et al. ACCF/AHA 2009 expert consensus document on pulmonary hypertension: a report of the American College of Cardiology Foundation Task Force on Expert Consensus Documents and the American Heart Association: developed in collaboration with the American College of Chest Physicians, American Thoracic Society, Inc., and the Pulmonary Hypertension Association. Circulation 2009; 119: 2250-2294.

25 Galiè N, Hoeper MM, Humbert M, et al. Guidelines for the diagnosis and treatment of pulmonary hypertension: the Task Force for the Diagnosis and Treatment of Pulmonary Hypertension of the European Society of Cardiology (ESC) and the European Respiratory Society (ERS), endorsed by the International Society of Heart and Lung Transplantation (ISHLT). Eur Heart J 2009; 30: 2493-2537.

26 O'Callaghan DS, Dorfmuller P, Jaïs X, et al. Pulmonary venoocclusive disease: the bête noire of pulmonary hypertension in connective tissue diseases? Presse Med 2011; 40: e65-e78.

27 Chang B, Schachna L, White B, et al. Natural history of mildmoderate pulmonary hypertension and the risk factors for severe pulmonary hypertension in scleroderma. J Rheumatol 2006; 33: 269-274.

28 Schachna L, Wigley FM, Chang B, et al. Age and risk of pulmonary arterial hypertension in scleroderma. Chest 2003; 124: 2098-2104. 
29 Plastiras SC, Karadimitrakis SP, Kampolis C, et al. Determinants of pulmonary arterial hypertension in scleroderma. Semin Arthritis Rheum 2007; 36: 392-396.

30 Scorza R, Caronni M, Bazzi S, et al. Post-menopause is the main risk factor for developing isolated pulmonary hypertension in systemic sclerosis. Ann N Y Acad Sci 2002; 966: 238-246.

31 Beretta L, Caronni M, Origgi L, et al. Hormone replacement therapy may prevent the development of isolated pulmonary hypertension in patients with systemic sclerosis and limited cutaneous involvement. Scand J Rheumatol 2006; 35: 468-471.

32 Steen V, Medsger TA Jr. Predictors of isolated pulmonary hypertension in patients with systemic sclerosis and limited cutaneous involvement. Arthritis Rheum 2003; 48: 516-522.

33 Coral-Alvarado P, Rojas-Villarraga A, Latorre MC, et al. Risk factors associated with pulmonary arterial hypertension in Colombian patients with systemic sclerosis: review of the literature. J Rheumatol 2008; 35: 244-250.

34 Chandra S, Shah SJ, Thenappan T, et al. Carbon monoxide diffusing capacity and mortality in pulmonary arterial hypertension. J Heart Lung Transplant 2010; 29: 181-187.

35 Allanore Y, Borderie D, Avouac J, et al. High N-terminal pro-brain natriuretic peptide levels and low diffusing capacity for carbon monoxide as independent predictors of the occurrence of precapillary pulmonary arterial hypertension in patients with systemic sclerosis. Arthritis Rheum 2008; 58: 284-291.

36 Nagaya N, Nishikimi T, Okano $Y$, et al. Plasma brain natriuretic peptide levels increase in proportion to the extent of right ventricular dysfunction in pulmonary hypertension. J Am Coll Cardiol 1998; 31: 202-208.

37 Williams MH, Handler CE, Akram R, et al. Role of N-terminal brain natriuretic peptide (NT-proBNP) in scleroderma-associated pulmonary arterial hypertension. Eur Heart J 2006; 27: 1485-1494.

38 Hesselstrand R, Wildt M, Ekmehag B, et al. Survival in patients with pulmonary arterial hypertension associated with systemic sclerosis from a Swedish single centre: prognosis still poor and prediction difficult. Scand J Rheumatol 2011; 40: 127-132.

39 Mathai SC, Bueso M, Hummers LK, et al. Disproportionate elevation of $\mathrm{N}$-terminal pro-brain natriuretic peptide in scleroderma-related pulmonary hypertension. Eur Respir J 2010; 35: 95-104.

40 Simeoni S, Lippi G, Puccetti A, et al. N-terminal pro-BNP in sclerodermic patients on bosentan therapy for PAH. Rheumatol Int 2008; 28: 657-660.

41 Vachiéry JL, Coghlan G. Screening for pulmonary arterial hypertension in systemic sclerosis. Eur Respir Rev 2009; 18: 162-169.

42 Vonk MC, Sander MH, van den Hoogen FH, et al. Right ventricle Tei-index: a tool to increase the accuracy of non-invasive detection of pulmonary arterial hypertension in connective tissue diseases. Eur J Echocardiogr 2007; 8: 317-321.

43 Serra W, Chetta A, Santilli D, et al. Echocardiography may help detect pulmonary vasculopathy in the early stages of pulmonary artery hypertension associated with systemic sclerosis. Cardiovasc Ultrasound 2010; 8: 25.

44 Schreiber BE, Valerio CJ, Keir GJ, et al. Improving the detection of pulmonary hypertension in systemic sclerosis using pulmonary function. Arthritis Rheum 2011; [Epub ahead of print DOI: 10.1002/ art.30535]

45 Meune C, Avouac J, Airò P, et al. Prediction of pulmonary hypertension related to systemic sclerosis by an index based on simple clinical observations. Arthritis Rheum 2011; 63: 2790-2796.

46 Arunthari V, Burger CD, Lee AS. Correlation of pulmonary function variables with hemodynamic measurements in patients with pulmonary arterial hypertension. Clin Respir J 2011; 5: 35-43.

47 Delcour KS, Singla A, Jarbou M, et al. Does reduced lung diffusing capacity for carbon monoxide predict the presence of pulmonary hypertension? Am J Med Sci 2010; 340: 54-59.

48 Cavagna L, Caporali R, Klersy C, et al. Comparison of brain natriuretic peptide (BNP) and NT-proBNP in screening for pulmonary arterial hypertension in patients with systemic sclerosis. J Rheumatol 2010; 37: 2064-2070.

49 Chung L, Liu J, Parsons L, et al. Characterization of connective tissue disease-associated pulmonary arterial hypertension from REVEAL: identifying systemic sclerosis as a unique phenotype. Chest 2010; 138: 1383-1394.

50 Hesselstrand R, Scheja A, Shen GQ, et al. The association of antinuclear antibodies with organ involvement and survival in systemic sclerosis. Rheumatology (Oxford) 2003; 42: 534-540.

51 Aggarwal R, Lucas M, Fertig N, et al. Anti-U3 RNP autoantibodies in systemic sclerosis. Arthritis Rheum 2009; 60: 1112-1118.

52 Mitri GM, Lucas M, Fertig N, et al. A comparison between antiTh/To- and anticentromere antibody-positive systemic sclerosis patients with limited cutaneous involvement. Arthritis Rheum 2003; 48: 203-209.

53 Pendergrass SA, Hayes E, Farina G, et al. Limited systemic sclerosis patients with pulmonary arterial hypertension show biomarkers of inflammation and vascular injury. PLoS One 2010; 5: e12106.

54 Christmann RB, Hayes E, Pendergrass S, et al. Interferon and alternative activation of monocyte/macrophages in systemic sclerosis-associated pulmonary arterial hypertension. Arthritis Rheum 2011; 63: 1718-1728.

55 Khanna D, Saggar R, Furst DE, et al. Predictive value of noninvasive tests for the diagnosis of scleroderma-associated pulmonary hypertension: PHAROS registry. Arthritis Rhem 2010; 62: Suppl. 10, 729.

56 ClinicalTrials.gov. Early, simple and reliable detection of pulmonary arterial hypertension (PAH) in systemic sclerosis (SSc) (DETECT). NCT00706082. http://clinicaltrials.gov/ct2/show/ NCT00706082?term $=$ NCT00706082\&rank=1 Date last updated: July 8, 2011. Date last accessed: July 11, 2011. 